\title{
PRESERVATION OF CLOSURE IN A LOCALLY CONVEX SPACE. I
}

BY

\author{
I. BRODSKY
}

\begin{abstract}
This paper is concerned with the lifting of the closures of sets. If $H$ is a topological vector space, $G$ a subspace and $A$ closed in $G$ for the induced topology, under what conditions on $A$ in $G$ is it true that the closure of $A$ is preserved in $H$, i.e., $\boldsymbol{A}$ is closed in $\boldsymbol{H}$ ? In this paper a fundamental lifting proposition is proved.

'Preservation of closure' will prove to be a fruitful technique in obtaining some interesting results in the theory of locally convex spaces. Using this technique, we will first show when closure is equivalent to completeness. Then we will prove a generalization to locally convex spaces of the classical Heine-Borel Theorem for Euclidean $n$-space. Generalizing a result of Petunin, we will also give some necessary and sufficient conditions on semireflexivity. Finally, we will give a necessary and sufficient condition for the sum of two closed subspaces to be closed.
\end{abstract}

Introduction. An interesting but not well-known result of Y. I. Petunin [2, pp. $1160-1162]$ is the following: Let $E$ be a Banach space and $S$ its unit ball. Then $E$ is reflexive if and only if $S$ is closed in every Hausdorff locally convex topology on $E$ that is weaker than the initial topology of $E$. The proof Petunin has given of his original theorem relies heavily on the concept of a characteristic of a subspace. However, we have found that the essence of Petunin's theorem does not depend upon the concept of a characteristic nor on boundedness nor on convexity. It will be seen that linearity is what makes Petunin's theorem work.

The form which Petunin's theorem now assumes gives little insight. Let us view Petunin's theorem from a different perspective. Reflexivity of $E$ is equivalent to having $S$ weakly compact. Also, $S$ is weakly compact if and only if $S$ is $\sigma\left(E^{\prime \prime}, E^{\prime}\right)$ closed in $E^{\prime \prime}$. Therefore, Petunin's theorem can be restated as follows: The unit ball $S$ is $\sigma\left(E^{\prime \prime}, E^{\prime}\right)$-closed in $E^{\prime \prime}$ if and only if $S$ is closed in every Hausdorff locally convex topology on $E$ that is weaker than the initial topology of $E$. It is this perspective that will prove to be very fruitful, in particular, in generalizing Petunin's theorem to arbitrary locally convex spaces.

We wish now to abstract the above situation. Let $H$ be a topological vector space and $G$ a subspace. Let $A$ be a proper subset of $G$. We wish to investigate under what conditions $A$ closed in $G$ implies $A$ closed in $H$. The reformulation of Petunin's theorem suggests that we look for a certain class of linear topologies on $G$

Received by the editors December 27, 1976 and, in revised form, March 16, 1977.

AMS (MOS) subject classifications (1970). Primary 46A99, 46-02; Secondary 46A05, 46A20, 46A25.

Key words and phrases. Preservation of closure, locally convex, completeness, reflexivity, semireflexivity, lifting of closures of sets.

${ }^{1}$ This research was supported partly by a National Science Foundation Fellowship. 
in which $A$ is closed. In Proposition 1, we isolate this class of linear topologies to insure the preservation of closure of $A$. We also obtain much more. This paper, in general, is an application of Proposition 1 and similar results to characterizations of various notions in topological vector space theory, e.g., completeness, compactness, semireflexivity.

In this paper, we will pursue the previously-stated problem in the following framework.

Definition 1. Let $X$ and $H$ be linear spaces with $\langle X, H\rangle$ a dual system. Let $G$ be a subspace of $H$ with $\langle G, X\rangle$ a paired (dual) system for the bilinear form from $\langle X, H\rangle$. If this is the case, we say that $\langle G, X, H\rangle$ is a triple (in duality).

Notation. If $\langle G, X, H\rangle$ is a triple and $A, B, C$ are subsets of $G, X, H$ respectively, we will write

(a) $A^{0}$ for the polar of $A$ in $X$,

(b) $C^{0}$ for the polar of $C$ in $X$,

(c) $B^{0}$ for the polar of $B$ in $G$,

(d) $B^{0}$ for the polar of $B$ in $H$.

A similar notation applies to the perpendicular, $\perp$, and subspaces.

Since every weakly dense subspace of the dual of a locally convex space gives a distinct Hausdorff locally convex topology on the base space, the following definition isolates two important classes of Hausdorff locally convex topologies.

Notation. If $G$ is a topological vector space with linear topology $\tau$, written $G[\tau]$ or $(G, \tau)$, and $A \subseteq D \subseteq G$ then the closure of $A$ in $D$ for $\tau$ will be denoted by $\tau \mid D-\mathrm{Cl}(A)$.

Definition 2. Let $\langle G, X, H\rangle$ be a triple. Define $\delta(G, X, H)=\{F: F$ is a subspace of $X, X=\sigma(X, G)-\mathrm{Cl}(F)$ and $F=\sigma(X, H)-\mathrm{Cl}(F)\}$. We also define $\mathscr{F}(G, X, H)=\left\{F: F \in \mathcal{S}(G, X, H)\right.$ and $F^{\perp}$ is finite dimensional in $\left.H\right\}$. We will write simply $\mathcal{S}$, respectively $\mathcal{F}$, if the triple is clear from the context.

The following elementary lemma does not seem to be in any of the literature. In what follows, $\operatorname{sp}\left(x, \ldots, x_{n}\right)$ denotes the linear span of $x_{1}, \ldots, x_{n}$.

Lemma 1. Let $\langle G, X\rangle$ be a paired system. Let $A$ be an absolutely convex $\sigma(G, X)$-closed subset and $H$ a finite-dimensional subspace of $G$. Then $A+H$ is an absolutely convex $\sigma(G, X)$-closed set.

Proof. It is sufficient to prove this lemma for $H=\operatorname{sp}(x), x \in G$. We may assume there exists a $y \in A^{0}$ such that $\langle x, y\rangle \neq 0$. Let $\left(a_{\lambda}+d_{\lambda} x\right) \in A+\operatorname{sp}(x)$ be a net, where $d_{\lambda}$ are scalars, and $z \in G$ such that $a_{\lambda}+d_{\lambda} x \rightarrow z$ for $\sigma(G, X)$. Since $\left(a_{\lambda}\right) \subseteq A=\left(A^{0}\right)_{0}$, there is a subnet of scalars $\left(\left\langle a_{\mu}, y\right\rangle\right)$ such that $\left\langle a_{\mu}, y\right\rangle \rightarrow r$, some scalar. Since $\langle x, y\rangle \neq 0$, it is easy to show there exists a scalar $c$ such that $a_{\mu} \rightarrow z-c x$ for $\sigma(G, X)$. This implies $z \in A+\operatorname{sp}(x)$.

Before we state and prove Proposition 1, we must make the following important observation. Let $\langle G, X, H\rangle$ be a triple. Let $B$ be a subset of $G, F$ a subspace of $X$ and $D$ a subset of $H$ containing $B$. Choose $g \in B$ and $h \in D$ such that $\langle f, g\rangle=$ $\langle f, h\rangle$ for all $f \in F$. If $A$ is a subset of $B$, then $g \in \sigma(B, F)-\mathrm{Cl}(A)$ if and only if $h \in \sigma(D, F)-\mathrm{Cl}(A)$, where, if $W \subseteq G, \sigma(W, F) \equiv \sigma(G, F) \mid W$. 
Proposition 1. Let $\langle G, X, H\rangle$ be a triple and $A \subset G$. If $\cup\{\sigma(G, F)-\mathrm{Cl}(A)$ : $F \in \mathscr{F}\} \subset G$, then for all $F \in \mathscr{F}, \sigma(H, F)-\mathrm{Cl}(A)=\sigma(G, F)-\mathrm{Cl}(A)+F^{\perp}$. In particular, $\sigma(G, X)-\mathrm{Cl}(A)=\sigma(H, X)-\mathrm{Cl}(A)$. Conversely, if $A$ is an absolutely convex $\sigma(H, X)$-closed subset of $G$, then for all $F \in \mathcal{F}, \sigma(G, F)-\mathrm{Cl}(A)=A$.

Proof. Let $F \in \mathcal{F}, h \in \sigma(H, F)-\mathrm{Cl}(A)$ and $B=\bigcup\{\sigma(G, F)-\mathrm{Cl}(A): F \in$ $\mathscr{F}\}$. Choose $g \in G \sim B$. Consider $N_{F}(h-g)$, the null space of $h-g$ restricted to $F$. If $\left.h\right|_{F}=\left.g\right|_{F}$, by the prior observation, $g \in \sigma(G, F)-\mathrm{Cl}(A)$ if and only if $h \in \sigma(H, F)-\mathrm{Cl}(A)$. Therefore, $h \in \sigma(H, F)-\mathrm{Cl}(A)$ implies $g \in \sigma(G, F)-$ $\mathrm{Cl}(A) \subseteq B$. Hence $g \in B$. This is a contradiction. Therefore, $N_{F}(h-g)$ is a hyperplane in $F$.

Case I. $N_{F}(g-h)$ is $\sigma(F, G)$-closed. Then $\left.(h-g)\right|_{F}$ is $\sigma(F, G)$-continuous. Since $\left.(h-g)\right|_{F}=\left.h\right|_{F}-\left.g\right|_{F}$ and $\left.g\right|_{F}$ are $\sigma(F, G)$-continuous, this $\left.h\right|_{F}$ is $\sigma(F, G)$ continuous. Since $F$ is $\sigma(X, G)$-dense, there exists a unique $\hat{h} \in G$ such that $\left.\hat{h}\right|_{F}=\left.h\right|_{F}$. Therefore, $\hat{h} \in G, h \in \sigma(H, F)-\mathrm{Cl}(A)$ and $\left.\hat{h}\right|_{F}=\left.h\right|_{F}$ imply $\hat{h} \in$ $\sigma(G, F)-\mathrm{Cl}(A)$. Since $\left.\hat{h}\right|_{F}=\left.h\right|_{F}$ implies $h-\hat{h} \in F^{\perp}$, we finally obtain $h \in \hat{h}+$ $F^{\perp} \subseteq \sigma(G, F)-\mathrm{Cl}(A)+F^{\perp}$.

Case II. $N_{F}(h-g)$ is $\sigma(F, G)$-dense. Therefore, $N_{F}(h-g) \in \mathcal{F}$. Since $h \in$ $\sigma(H, F)-\mathrm{Cl}(A) \subseteq \sigma\left(H, N_{F}(h-g)\right)-\mathrm{Cl}(A), g \in \sigma\left(G, N_{F}(h-g)\right)-\mathrm{Cl}(A) \subseteq B$. Hence $g \in B$. This is a contradiction.

We have shown that Case I must always hold. Therefore $\sigma(H, F)-\mathrm{Cl}(A) \subseteq$ $\sigma(G, F)-\mathrm{Cl}(A)+F^{\perp}$, for all $F \in \mathscr{F}$. Clearly, $\sigma(G, F)-\mathrm{Cl}(A)+F^{\perp} \subseteq \sigma(H, F)$ $-\mathrm{Cl}(A)$ for all $F \in \mathscr{F}$. Hence, for all $F \in \mathscr{F}, \sigma(H, F)-\mathrm{Cl}(A)=\sigma(G, F)-$ $\mathrm{Cl}(A)+F^{\perp}$.

That the first part of the proposition implies $\sigma(G, X)-\mathrm{Cl}(A)=\sigma(H, X)-$ $\mathrm{Cl}(A)$ follows from the fact that $X \in \mathcal{F}$.

Conversely, fix $F \in \mathscr{F}$. To show $A$ is $\sigma(G, F)$-closed, first let $\phi$ be the canonical map $H \rightarrow H / F^{\perp}$. Since $F=\left(F^{\perp}\right)_{\perp}$, we have by Schaefer [3, Corollary 1 of Theorem 1, p. 135] that the quotient topology on $H / F^{\perp}$ derived from $\sigma(H, X)$ is $\sigma\left(H / F^{\perp}, F\right)$. By Lemma $1, A+F^{\perp}$ is $\sigma(H, X)$-closed. Therefore $\phi(A)$ is $\sigma\left(H / F^{\perp}, F\right)$-closed. Since $G$ is algebraically embedded in $H / F^{\perp}$, we have that $A$ is $\sigma(G, F)$-closed.

Remark. In the first part of Proposition 1, the only place we used the fact that $F^{\perp}$ is of finite dimension in $H$ for $F \in \mathscr{F}(G, X, H)$ was in claiming that $N_{F}(h-g) \in \mathscr{F}(G, X, H)$. Therefore, we can obtain a result similar to the first part of Proposition 1 by replacing $\mathscr{F}(G, X, H)$ with $\mathcal{S}(G, X, H)$.

Remark. Using the same technique as in Proposition 1, we can prove the following similar result: Let $\langle G, X, H\rangle$ be triple with $B, D$ subsets of $G$ such that $B \subset D$ and $D$ is $\sigma(G, X)$-closed. If $B$ is $\sigma(D, F)$-closed for every $F \in \mathscr{F}$, then $B$ is $\sigma(H, X)$-closed.

We will now apply Proposition 1 to obtain an interesting equivalence between closure and completeness. However, before we state Theorem 1, we need the following well-known results. We simply state them.

(A) Let $X$ be a linear space. If $F$ is a subspace of $X$, then $F$ is $\sigma\left(X, X^{*}\right)$-closed.

(B) Let $G$ be a locally convex space with topology $\tau$. Let $A$ be a subset of $G$ and 
$X=G^{\prime}$. If $A$ is $\sigma(G, X)$-complete, then $A$ is $\tau$-complete.

In the following theorem H.L.C.T. will stand for Hausdorff Locally Convex Topology.

THeOREM 1. Let $G$ be a locally convex space with topology $\tau$. Let $A$ be a properly contained in $G$. Then the following are equivalent.

(a) For every H.L.C.T. $\pi$ on $G$ such that $\pi<\tau, A$ is $\pi$-closed.

(b) For every H.L.C.T. $\pi$ on $G$ such that $\pi<\tau, A$ is $\pi$-complete.

Proof. Let $X=G^{\prime}$. By (B) it is sufficient to prove that the following are equivalent.

(a') For every H.L.C.T. $\pi$ on $G$ such that $\pi<\sigma(G, X), A$ is $\pi$-closed.

(b') For every H.L.C.T. $\pi$ on $G$ such that $\pi<\sigma(G, X), A$ is $\pi$-complete.

$\left(\mathrm{a}^{\prime}\right) \Rightarrow\left(\mathrm{b}^{\prime}\right)$. Let $\pi$ be a H.L.C.T. on $G$ such that $\pi<\sigma(G, X)$ and $T=G[\pi]^{\prime} \subseteq X$. Let $S \subseteq T$ be such that $\sigma(G, S)$ is an H.L.C.T. on $G$ weaker than $\sigma(G, T)$. Therefore $\sigma(G, S)<\sigma(G, X)$ and thus $A$ is $\sigma(G, S)$-closed. Using (A), we have shown that for every $F \in \mathscr{F}\left(G, T, T^{*}\right), A$ is $\sigma(G, F)$-closed. Therefore, since $\left\langle G, T, T^{*}\right\rangle$ is a triple (in duality), we have by Proposition 1 that $A$ is $\sigma\left(T^{*}, T\right)$ closed. The completion of $(G, \sigma(G, T))$ is $\left(T^{*}, \sigma\left(T^{*}, T\right)\right)$. Therefore, $A$ is $\sigma(G, T)$ complete and by (B) $A$ is $\pi$-complete.

$\left(\mathrm{b}^{\prime}\right) \Rightarrow\left(\mathrm{a}^{\prime}\right)$. This is obvious.

The classical Heine-Borel Theorem for Euclidean $n$-space states that every closed and bounded subset is (weakly) compact. The Heine-Borel Theorem does not hold in general locally convex spaces. The following theorem is an interesting generalization of this result.

Notation. Let $G$ be a locally convex space with topology $\tau$. We will write $\mathfrak{R}(G[\tau])$ for a fundamental system of closed bounded absolutely convex subsets of $G[\tau]$ and $\mathcal{Q}(G[\tau])$ for a fundamental system of 0-neighborhoods, consisting of closed convex sets.

Before we can state Theorem 2, we need the following setting. Let $E$ be a locally convex space with topology $\tau$ and $K(E)=\{f: f$ is continuous from $E$ into $\mathbf{K}$, the scalars, and bounded on $M, M \in \mathfrak{R}\}$. Since $E$ is completely regular as a topological space, $K(E)$ contains a sufficient number of functions to recover the original topology of $E$, that is, the inductive topology on $E$ generated by the family $K(E)$ is $\tau$.

Let $L$ be a linear subspace of $K(E)$ such that $E^{\prime} \subseteq L \subseteq K(E)$. Consider $E$ as linear forms on $L$, i.e., $E \hookrightarrow L^{*}$. From the dual system $\langle\operatorname{sp}(E), L\rangle$, where $\operatorname{sp}(E)$ denotes the linear span of $E$ in $L^{*}$, we can form the locally convex space $T(L)=(\operatorname{sp}(E), \sigma(\operatorname{sp}(E), L))$.

If $L=E^{\prime}$, then $T(L)=\left(E, \sigma\left(E, E^{\prime}\right)\right)$. If $L=K(E)$, we have in general altered the linear structure of $E$, i.e., $E$ is not necessarily a linear subspace of $K(E)^{*}$. However, if $L=K(E), E[\tau]$ is topologically embedded in $T(L)$. For arbitrary $L$, if $M \subseteq E$ then $M$ is bounded in $T(L)$ if and only if $M$ is bounded in $E[\tau]$.

We will write $T$ instead of $T(L)$ if $L$ is clear from the context. 
THEOREM 2. Let $E$ be a locally convex space with topology $\tau$ and $M$ a bounded subset of $E$. Let $L$ be a linear subspace of $K(E)$ such that $E^{\prime} \subseteq L$ and $\mathcal{F}=$ $\mathscr{F}\left(T, T^{\prime}, T^{\prime \prime}\right)$. Then the following are equivalent.

(a) For all $F \in \mathscr{F}, M$ is $\sigma(T, F)$-closed.

(b) $M$ is $\sigma\left(T, T^{\prime}\right)$-compact. In particular, if $L=E^{\prime}, M$ is $\sigma\left(E, E^{\prime}\right)$-compact and if $L=K(E), M$ is $\tau$-compact.

Proof. (a) $\Rightarrow(\mathrm{b})$. Since $\left\langle T, T^{\prime}, T^{\prime \prime}\right\rangle$ is a triple in duality, we have by Proposition 1 that $M$ is a $\sigma\left(T^{\prime \prime}, T^{\prime}\right)$-closed subset of $T$. By the Bourbaki-Alaoglu Theorem $M^{00}$ is $\sigma\left(T^{\prime \prime}, T^{\prime}\right)$-compact. Since $M \subseteq M^{00}, M$ is a $\sigma\left(T^{\prime \prime}, T^{\prime}\right)$-compact subset of $T$ and hence $\sigma\left(T, T^{\prime}\right)$-compact. In particular, if $L=E^{\prime}, M$ is $\sigma\left(E, E^{\prime}\right)$-compact and if $L=K(E)$, by the complete regularity of $E, M$ is $\tau$-compact.

(b) $\Rightarrow\left(\right.$ a). Fix $F \in \mathscr{F}$. Since $M$ is $\sigma\left(T, T^{\prime}\right)$-compact, $M$ is $\sigma(T, F)$-compact and hence $\sigma(T, F)$-closed.

REMARK. If $X$ is a completely regular topological space, we can employ the previous technique in the following setting to obtain a necessary and sufficient condition for the compactness of $X$ : Let $B(X)=\{f: f$ is a bounded real-valued continuous function on $X\}$. As before, we consider $X$ as linear forms on $B(X)$ and obtain the locally convex space $\left(\operatorname{sp}(X), \sigma\left(\operatorname{sp}(X), B(X)^{*}\right)\right)$, where $\operatorname{sp}(X)$ is the linear span of $X$ in $B(X)^{*}$.

We are now prepared to give a generalization of Petunin's Theorem [2] on Banach spaces to arbitrary locally convex spaces. Our generalization will be in two directions; in one, the unit ball will be replaced by $\mathfrak{N}$ and, in the other, by a proper bornivorous set.

THEOREM 3. Let $E$ be a locally convex space with topology $\tau$. If $\mathscr{F}=$ $\mathscr{F}\left(E, E^{\prime}, E^{\prime \prime}\right)$, then the following are equivalent.

(a) $E$ is semireflexive.

(b) For every $M \in \mathfrak{N}$ and H.L.C.T. $\rho$ on $E$ such that $\rho<\tau, M$ is $\rho$-closed.

(c) For every $M \in \mathfrak{N}$ and $F \in \mathscr{F}, M$ is $\sigma(E, F)$-closed.

(d) There exists a proper bornivorous subset $B$ of $E$ such that for every $F \in \mathcal{F}, B$ is $\sigma(E, F)$-closed.

(e) For every $M \in \mathfrak{N}$ and $F \in \mathcal{F}, \sigma(M, F)=\sigma\left(M, E^{\prime}\right)$.

(f) There exists a bornivorous barrel $B$ of $E[\tau]$ such that for every $F \in \mathscr{F}$, $\sigma(B, F)=\sigma\left(B, E^{\prime}\right)$.

(g) $\cap\{F: F \in \mathscr{F}\} \neq(0)$.

Proof. In the course of the proof we will make free use of the following well-known criteria for semireflexivity. The locally convex space $E$ is semireflexive if and only if every $M \in \mathfrak{N}$ is $\sigma\left(E, E^{\prime}\right)$-compact. By the Bourbaki-Alaoglu theorem, for every $M \in \mathfrak{T}, \sigma\left(E^{\prime \prime}, E^{\prime}\right)-\mathrm{Cl}(M)=M^{00}$ is $\sigma\left(E^{\prime \prime}, E^{\prime}\right)$-compact. Therefore, to show $E$ is semireflexive it is necessary and sufficient to have every $M \in \mathfrak{R}, \sigma\left(E^{\prime \prime}, E^{\prime}\right)$-closed.

(a) $\Rightarrow(\mathrm{b})$. Since $E=E^{\prime \prime}$, it is clear every $M \in \mathfrak{N}$ is $\sigma\left(E, E^{\prime}\right)$-compact. If $S=E[\rho]^{\prime} \subseteq E^{\prime}$, every $M \in \Re$ is $\sigma(E, S)$-closed and thus, by convexity, $\rho$-closed.

(b) $\Rightarrow$ (c). This is obvious. 
(c) $\Rightarrow$ (a). By Theorem 2, every $M \in \mathscr{T}$ is $\sigma\left(E, E^{\prime}\right)$-compact and thus $E$ is semireflexive.

If $E$ is semireflexive, the $\sigma\left(E^{\prime}, E\right)$-closure equals the $\sigma\left(E^{\prime}, E^{\prime \prime}\right)$-closure. Clearly, this implies $\mathscr{F}=\left\{E^{\prime}\right\}$. In this case, i.e., if $E$ is semireflexive, conditions (d)-(g) are all trivial. Therefore, to finish the proof it is sufficient to show conditions (d)-(g) in turn imply that $E$ is semireflexive.

(d) $\Rightarrow$ (a). By Proposition 1, we have that $B$ is $\sigma\left(E^{\prime \prime}, E^{\prime}\right)$-closed. Since $B$ absorbs $\sigma\left(E, E^{\prime}\right)$-bounded subsets, we obtain every $M \in \mathfrak{N}$ is $\sigma\left(E^{\prime \prime}, E^{\prime}\right)$-closed.

(e) $\Rightarrow$ (a). Clearly $M / 2 \subset M$ and $M / 2$ is $\sigma\left(M, E^{\prime}\right)$-closed. Therefore, $M / 2$ is $\sigma(M, F)$-closed, for every $F \in \mathcal{F}$. By the remark following Proposition 1, this implies $M / 2$ is $\sigma\left(E^{\prime \prime}, E^{\prime}\right)$-closed and thus $M$ is $\sigma\left(E^{\prime \prime}, E^{\prime}\right)$-closed.

(f) $\Rightarrow(\mathrm{a})$. If $B=E, \sigma\left(E, E^{\prime}\right)=\sigma(E, F)$, for every $F \in \mathscr{F}$ and thus $\cap\{F$ : $F \in \mathscr{F}\}=E^{\prime}$, which will be shown to imply (a) in the next part. Therefore, we may assume $B \subset E$. Clearly we then have $B / 2 \subset B$. The remainder is a repitition of parts of $(e) \Rightarrow(a)$ and $(d) \Rightarrow(a)$.

(g) $\Rightarrow$ (a). If $0 \neq y \in \cap\{F: F \in \mathcal{F}\}$, the proper bornivorous subset $\{Y\}_{0}$ of $E$ is $\sigma(E, F)$-closed for every $F \in \mathcal{F}$. By (d), we obtain $E$ is semireflexive.

COROLlary 1. Let $E$ be a locally convex space. Then $E$ is reflexive if and only if $E$ is infrabarrelled and satisfies any of the conditions (b) $-(\mathrm{g})$.

Corollary 2 (PetUnin). Let E be a normed linear space with topology $\tau$. Then $E$ is reflexive if and only if its unit ball is closed in $E$ for every H.L.C.T. on E weaker than $\tau$.

Notation. Let $G$ be a linear space, $A$ a subset of $G, F$ a subspace of $G$ and $x \in G$. We will sometimes write $A / F$ for $\phi(A)$ and $\hat{x}$ for $\phi(x)$, where $\phi$ is the canonical map from $G$ onto $G / F$.

We will now give a necessary and sufficient condition for the sum of two closed subspaces to be closed. We will need the following lemma.

Lemma 2. Let $\langle G, X\rangle$ be a dual system and $F_{1}, F_{2}$ two $\sigma(G, X)$-closed subspaces such that $F_{1}+F_{2}$ is properly contained in $G$. Let $\phi$ be the canonical map from $G$ onto $G / F_{1} \cap F_{2}$ and $x \in G \sim\left(F_{1}+F_{2}\right)$. Then

(a) $\left\langle\phi\left(F_{1} \oplus \operatorname{sp}(x)\right),\left(F_{1} \cap F_{2}\right)^{\perp}, \phi(G)\right\rangle$ is a triple and from this we obtain the triple

$$
\left\langle\phi\left(F_{1} \oplus \operatorname{sp}(x)\right) / \phi\left(F_{2}\right), \phi\left(F_{2}\right)_{\perp}, \phi(G) / \phi\left(F_{2}\right)\right\rangle .
$$

(b) The dual space $\left(F_{1} \cap F_{2}\right)^{\perp}$ does not identify $\phi(x)$ with any element of $\phi\left(F_{1}\right)$, i.e., $\phi\left(F_{1}\right)$ is a proper subspace of $\left(\phi\left(F_{1} \oplus \operatorname{sp}(x)\right), \sigma\left(\phi\left(F_{1} \oplus \operatorname{sp}(x)\right),\left(F_{1} \cap F_{2}\right)^{\perp}\right)\right)$.

(c) The dual space $\phi\left(F_{2}\right)_{\perp}$ does not identify $\hat{\alpha}, \alpha=\phi(x)$, with any element of $\phi\left(F_{1}\right) / \phi\left(F_{2}\right)$, i.e., $\phi\left(F_{1}\right) / \phi\left(F_{2}\right)$ is a proper subspace of $\left(\phi\left(F_{1} \oplus \operatorname{sp}(x)\right) / \phi\left(F_{2}\right)\right.$, $\left.\sigma\left(\phi\left(F_{1} \oplus \operatorname{sp}(x)\right) / \phi\left(F_{2}\right), \phi\left(F_{2}\right) \perp\right)\right)$.

Proof. (a) It is sufficient to show that $\left\langle\left(F_{1} \cap F_{2}\right)^{\perp}, \phi(G)\right\rangle$ and $\left\langle\phi\left(F_{2}\right)_{\perp}, \phi(G) / \phi\left(F_{2}\right)\right\rangle$ are dual systems. For the first, since $F_{1} \cap F_{2}$ is $\sigma(G, X)$ closed, it is clear that $\left\langle\left(F_{1} \cap F_{2}\right)^{\perp}, \phi(G)\right\rangle$ is the dual system. It also follows that 
the $\sigma(G, X)$-quotient topology on $\phi(G)$ is $\sigma\left(\phi(G),\left(F_{1} \cap F_{2}\right)^{\perp}\right)$. Therefore, since $F_{2}+F_{1} \cap F_{2}=F_{2}$ is $\sigma(G, X)$-closed, $\phi\left(F_{2}\right)$ is $\sigma\left(\phi(G),\left(F_{1} \cap F_{2}\right)^{\perp}\right)$-closed. Therefore, from the dual system $\left\langle\left(F_{1} \cap F_{2}\right)^{\perp}, \phi(G)\right\rangle$, we obtain the dual system $\left\langle\phi\left(F_{2}\right)_{\perp}, \phi(G) / \phi\left(F_{2}\right)\right\rangle$

(b) Suppose there exists an $f \in F_{1}$ such that $\langle\phi(f)-\phi(x), y\rangle=0$, for all $y \in\left(F_{1} \cap F_{2}\right)^{\perp}$. Since $\left(F_{1} \cap F_{2}\right)^{\perp}$ separates points of $\phi(G)$, this implies $\phi(f)=$ $\phi(x)$ and thus $x-f \in F_{1} \cap F_{2}$. Therefore $x \in F_{1}+F_{2}$. This is a contradiction.

(c) Apply the same proof as in (b) to $\hat{\alpha}, \phi\left(F_{1}\right) / \phi\left(F_{2}\right)$ and $\left\langle\phi\left(F_{2}\right)_{\perp}, \phi(G) / \phi\left(F_{2}\right)\right\rangle$.

THEOREM 4. If the setting is as in Lemma 2 then $F_{1}+F_{2}$ is $\sigma(G, X)$-closed if and only if for every $S \in \mathcal{F}=\mathscr{F}\left(\phi\left(F_{1} \oplus \operatorname{sp}(x)\right), \phi\left(F_{2}\right)_{\perp}, \phi(G) / \phi\left(F_{2}\right)\right), \phi\left(F_{1}\right)$ is $\sigma\left(\phi\left(F_{1} \otimes \mathrm{sp}(x)\right), S\right)$-closed.

Proof. Let us first observe that since $\phi\left(F_{2}\right)_{\perp}$ separates points of $\phi\left(F_{1} \oplus \operatorname{sp}(x)\right)$, we can identify $\phi\left(F_{1} \oplus \mathrm{sp}(x)\right)$ with its image in $\phi(G) / \phi\left(F_{2}\right)$, i.e., with $\phi\left(F_{1} \oplus \operatorname{sp}(x)\right) / \phi\left(F_{2}\right)$. Therefore $\mathscr{F}\left(\phi\left(F_{1} \oplus \operatorname{sp}(x)\right) / \phi\left(F_{2}\right), \phi\left(F_{2}\right)_{\perp}, \phi(G) / \phi\left(F_{2}\right)\right)=$ $\mathscr{F}\left(\phi\left(F_{1} \oplus \mathrm{sp}(x)\right), \phi\left(F_{2}\right)_{\perp}, \phi(G) / \phi\left(F_{2}\right)\right)$.

NeCESSITY. Fix $S \in \mathscr{F}$. We have the following sequence of locally convex spaces,

$$
\begin{gathered}
\sigma(G, X) \quad \sigma\left(\phi(G),\left(F_{1} \cap F_{2}\right)^{\perp}\right) \quad\left(\phi(G) / \phi\left(F_{2}\right), \phi\left(F_{2}\right)_{\perp}\right) \\
G \longrightarrow \phi(G) \longrightarrow \phi(G) / \phi\left(F_{2}\right),
\end{gathered}
$$

where each topology above its respective space is the quotient of the preceding topology. Therefore, $F_{1}+F_{2} \sigma(G, X)$-closed

$\Rightarrow F_{1}+F_{2}+F_{1} \cap F_{2}$ is $\sigma(G, X)$-closed,

$\Rightarrow \phi\left(F_{1}\right)+\phi\left(F_{2}\right)$ is $\sigma\left(\phi(G),\left(F_{1} \cap F_{2}\right)^{\perp}\right)$-closed,

$\Rightarrow \phi\left(F_{1}\right)$ is $\sigma\left(\phi(G) / \phi\left(F_{2}\right), \phi\left(F_{2}\right)_{\perp}\right)$-closed,

by our identification.

By Proposition 1 applied to $\mathscr{F}$, this implies $\phi\left(F_{1}\right)$ is $\sigma\left(\phi\left(F_{1} \oplus \operatorname{sp}(x)\right), S\right)$-closed.

SufficienCY. By Proposition $1, \phi\left(F_{1}\right)$ is $\sigma\left(\phi(G) / \phi\left(F_{2}\right), \phi\left(F_{2}\right)_{\perp}\right)$-closed and by the continuity of the quotient map we further obtain that $\phi\left(F_{1}\right)+\phi\left(F_{2}\right)$ is $\sigma\left(\phi(G), /\left(F_{1} \cap F_{2}\right)^{\perp}\right)$-closed. Again, by the continuity of the quotient map $\phi$, we have that $\phi^{-1}\left(\phi\left(F_{1}\right)+\phi\left(F_{2}\right)\right)=F_{1}+F_{2}+F_{1} \cap F_{2}$ is $\sigma(G, X)$-closed. Thus $F_{1}+$ $F_{2}$ is $\sigma(G, X)$-closed.

REMARK. If in Theorem $2, F_{1} \cap F_{2}=(0)$, then $\mathscr{F}$ becomes

$$
\mathscr{F}\left(F_{1} \oplus \operatorname{sp}(x),\left(F_{2}\right)_{\perp}, G /\left(F_{2}\right)\right) \text {. }
$$

\section{REFERENCES}

1. J. Horváth, Topological vector spaces and distributions. I, Addison-Wesley, Reading, Mass., 1966.

2. Y. Petunin, Criterion for reflexivity of a Banach space, Soviet Math. Dokl. 2 (1961), 1160-1162.

3. H. H. Schaefer, Topological vector spaces, Macmillan, New York, 1966.

Department of Mathematics, University of Puerto Rico, Rio Piedras, Puerto Rico 00931 